\title{
Bounds on Sectional Curvature and Interactions with Topology
}

\author{
Manuel Amann1
}

Accepted: 9 November 2020 / Published online: 17 November 2020

(C) The Author(s) 2020

\begin{abstract}
In this survey article we exemplarily illustrate implications of curvature assumptions on the topology of the underlying manifold. We shall mainly focus on sectional curvature and three different kinds of restrictions, namely on non-negative respectively on positive sectional curvature, as well as on two-sided curvature bounds.

We shall see that there are various implications on the side of topology, namely, for example, geometry having an impact on elementary invariants like the Euler characteristic or Betti numbers as well as on concepts from rational homotopy theory or index theory, and that there are connections to K-theory.

On our way of making these connections we shall draw on certain simplifications and tools like group actions or metrics with additional properties like geometric formality.
\end{abstract}

Mathematics Subject Classification (2010) Primary 53C20 · Secondary 57N65

Keywords Non-negative sectional curvature $\cdot$ Positive curvature $\cdot$ Two-sided curvature bounds $\cdot$ Rational homotopy $\cdot$ Symmetry $\cdot$ Geometric formality $\cdot$ Elliptic genus $\cdot$ K-theory $\cdot$ Soul theorem $\cdot$ Equivariant cohomology

\section{Introduction}

One of the main goals of Riemannian geometry is to understand the nature and behaviour of curvature. More concretely, this comprises the study of the following Janus-faced

\section{Amann}

url: http://www.uni-augsburg.de/de/fakultaet/mntf/math/prof/diff/team/dr-habil-manuel-amann/; manuel.amann@math.uni-augsburg.de

1 Institut für Mathematik, Differentialgeometrie, Universität Augsburg, Universitätsstraße 14, 86159 Augsburg, Germany 
Question - What implications on the topology of a manifold do bounds on curvature have?

- What topological obstructions exist against the existence of metrics with certain curvature bounds?

One aspect which makes these questions especially hard to answer is a passage from a local to a global viewpoint: Curvature is a locally defined concept, whereas, whatever encodes the topology of the manifold should be a global invariant.

It is the goal of this survey article to reflect on various aspects of these questions; in non-negative and positive curvature, as well as with two-sided curvature bounds. On the topological side we shall meet elementary invariants like the Euler characteristic and more advanced concepts from rational homotopy theory, index theory and K-theory. We also refer the reader to the survey article [2] which complements and extends this one especially for positively curved metrics; we tried to keep intersections as small as possible whilst preserving many different aspects of the interplay of curvature and topology.

The term "curvature" comes in many different versions and concretisations; there is not the curvature, but depending on purpose, goal, needed flexibility etc. different variants are considered. Most classically, and increasing in strength, scalar, Ricci and sectional curvature are to be mentioned. In this article, our focus will lie on sectional curvature, mainly on positive and non-negative sectional curvature as well as twosided bounds on sectional curvature. Accordingly, whenever we speak of "positively curved" or "non-negative curvature", etc., this always refers to the respective sign of the sectional curvature. In the following, let us quickly recall different classical curvature notions.

Let $\left(M^{n},\langle\cdot\rangle\right)$ be a Riemannian manifold, $p \in M$. The most rigid classical curvature concept (consequently containing most information), which then gives rise to the other ones, is sectional curvature. Sectional curvature depends on a point and a two-dimensional plane in the tangent space at the point. It describes the curvature of the manifold along this plane. In contrast, Ricci curvature only depends on the point and a direction, as it averages over the 2-planes extending this direction, and scalar curvature averages over all 2-planes at the point. Hence it is the weakest invariant of the three ones and can be computed from the knowledge of the sectional curvatures. Trivially, for example, positive (sectional) curvature implies positive scalar curvature.

More precisely, sectional curvature in $p$ of a plane $E$ spanned by an orthonormal basis $v, w \in T_{p} M$ is given by

$$
K(v, w)=\left\langle R_{v, w} w, v\right\rangle_{p}
$$

where $R$ denotes the Riemannian curvature tensor. Note that the sectional curvature $K(v, w)$ indeed only depends on the chosen plane. Contractions of sectional curvature lead to Ricci and scalar curvature, $\operatorname{ric}_{p}(v):=\sum_{i=1}^{n} K\left(e_{i}, v\right)$, and $\operatorname{scal}_{p}:=$ $\sum_{i=1}^{n} \operatorname{ric}_{p}\left(e_{i}\right)$ for an orthonormal basis $\left(e_{i}\right)_{1 \leq i \leq n}$. As already stated above, we shall speak of positive respectively non-negative (sectional) curvature etc., whenever in all points for all planes sectional curvature is positive respectively non-negative.

For a geometric interpretation of sectional curvature, one may view it locally as the Gaussian curvature of the surface defined by the chosen plane in $p$ under the exponential map. 

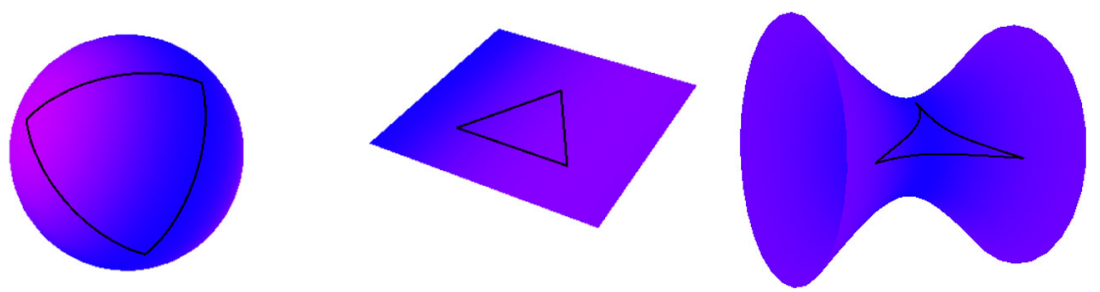

Fig. 1 Geodesic triangles, $K>0, K=0$ and $K<0$

The simplest cases to consider are those metrics of constant (sectional) curvature. Such a manifold is universally covered by either a round sphere, Euclidean or hyperbolic space-depending on the sign of its curvature.

This permits us to characterise curvature bounds in a metric way. For example, our manifold will be more curved than the standard sphere, i.e. it will have lower curvature bound 1, if all its geodesic triangles are "fat", i.e. have angle sum larger than $\pi$. Analogously, as we are used to, in flat space the angle sum is $\pi$. In negative curvature triangles are "thin" and have angle sum smaller $\pi$. For example, there will be an upper curvature bound of -1 if all geodesic triangles are thinner than corresponding triangles in the hyperbolic plane. (It can be shown that such "comparison triangles" with the same side lengths as the original triangles exist.)

Let us begin by reviewing some famous theorems dealing with implications of the various curvature notions. We begin with the weakest curvature notion, scalar curvature. Here, on the topological side we obtain an invariant from K-theory which obstructs the existence of a metric of positive scalar curvature in several cases.

Theorem (Gromov-Lawson, Stolz) Let $M$ be a compact simply-connected manifold of dimension at least 5 , which is not spin. Then $M$ admits a metric of positive scalar curvature. A simply-connected spin manifold of dimension at least 5 carries a metric of positive scalar curvature if and only if its $\alpha$-invariant is zero.

Recall that the $\alpha$-invariant is a spin cobordism invariant with values in $\mathrm{KO}^{*}(\mathrm{pt})$ and a K-theoretic refinement of the $\hat{A}$-genus, i.e. it agrees with it (up to factor) if the dimension of the manifold is divisible by four. Due to periodicity, the $\alpha$-invariant hence provides additional $\mathbb{Z}_{2}$-valued obstructions in case $\operatorname{dim} M \equiv 1,2 \bmod 8$ extending the $\mathbb{Z}$-valued obstruction of the $\hat{A}$-genus in degrees $\operatorname{dim} M \equiv 0 \bmod 4$. The $\hat{A}$-genus will appear later again.

As for Ricci curvature a famous theorem by Bonnet-Myers states that a compact manifold with positive Ricci curvature has a finite fundamental group-more generally, this holds true (and also compactness is implied) for a complete connected Riemannian manifold with a uniform lower Ricci bound. This is strengthened by Synge's theorem in the case of positive sectional curvature. Note however, that, as the famous example of the paraboloid illustrates, the non-existence of a uniform lower Ricci/sectional curvature bound may lead to non-compact manifolds of positive Ricci curvature. That is, in the case of the paraboloid (given as the graph $z=x^{2}+y^{2}$ ) Ricci curvature is strictly positive but tends to zero for large $x, y$; however, this surface is non-compact. 
In contrast, negative Ricci curvature has no strong implications.

Theorem (Lohkamp) Every manifold of dimension $\geq 3$ admits a metric of negative Ricci curvature.

Non-positive sectional curvature, however, is understood in

Theorem (Hadamard-Cartan) A simply-connected complete Riemannian manifold $\left(M^{n}, g\right)$ with non-positive sectional curvature is diffeomorphic to $\mathbb{R}^{n}$.

Hence, topology in this situation lies in the study of the fundamental group. We are, however, more interested in simply-connected spaces and non-negative curvature.

As a summary, we have discussed positive scalar curvature, negative Ricci and non-positive sectional curvature. What is certainly left open in this list of results is a topological characterisation of non-negatively and positively curved metrics. This will be the focus of the rest of the article.

\section{Non-negative and Positive Sectional Curvature - Examples, Obstructions, Conjectures}

If not stated differently, during the rest of the article our manifolds will be closed, i.e. compact without boundary, and simply-connected.

\subsection{Examples}

\subsubsection{Non-negative Curvature}

There are various examples of closed manifolds admitting metrics of non-negative sectional curvature. As for manifolds of positive sectional curvature the situation changes drastically. So it seems natural to first discuss non-negative curvature before we turn to positive curvature.

A major source of examples of non-negatively curved manifolds is constituted by compact Lie groups $G$ which we may always equip with a biinvariant metric-both left and right multiplication with elements from $G$ are isometries. Then it holds that sectional curvature

$$
K(X, Y)=\frac{1}{4}|[X, Y]|^{2} \geq 0
$$

(for $X, Y \in \mathfrak{g}$ orthonormal vectors from the Lie algebra of $G$ ) is non-negative (where $[\cdot, \cdot]$ denotes the Lie bracket). Note that due do the biinvariant metric and homogeneity it suffices to consider sectional curvature at the identity $e \in G$ with $T_{e} G \cong \mathfrak{g}$. Hence, sectional curvature obviously vanishes on maximal tori, i.e. on maximal connected abelian subgroups. Consequently, combining this with the classification of compact Lie groups, we easily derive that the only simply-connected compact Lie 
group admitting a biinvariant metric of positive curvature is $\mathbf{S p}(1) \cong \mathbf{S U}(2) \cong \mathbb{S}^{3}$ all the other ones (of dimension at least 2) have an at least 2-dimensional maximal torus.

Example 2.1 Given two non-negatively curved Riemannian manifolds $\left(M, g_{1}\right)$, ( $\left.N, g_{2}\right)$, the product metric $g_{1} \oplus g_{2}$ on $M \times N$ is non-negatively curved. However, it has flats (even if both $g_{1}$ and $g_{2}$ are positively curved), since the curvature in a plane spanned by a (unit-length) vector $X$ in the tangent space of $M$ and another one, $Y$, in the tangent space of $N$ is zero. This follows from

$$
K(X, Y)=R(X, Y, X, Y)=R_{1}(X, 0, X, 0)+R_{2}(0, Y, 0, Y)=0
$$

with the curvature tensors $R_{1}, R_{2}$ on $\left(M, g_{1}\right),\left(N, g_{2}\right)$. 回

This example actually sets the stage for one of the most prominent conjectures in Riemannian geometry. It is one out of two conjectures by Hopf which have spurred a lot of research in positive curvature. The second conjecture being more topological in nature will be presented later.

Conjecture 2.2 (Hopf) The manifold $\mathbb{S}^{2} \times \mathbb{S}^{2}$ does not possess any metric of positive sectional curvature.

This conjecture is settled within special classes of metrics. For example, it holds if the metric is invariant under an action of the circle group $\mathbb{S}^{1}$ as follows by a theorem of Hsiang-Kleiner (see Theorem 3.2), it is also known in the class of "formal metrics" (see Sect. 3.2, Theorem 3.8) by Bär, Kotschick. In general, however, this conjecture is widely open, and, clearly, replacing $\mathbb{S}^{2} \times \mathbb{S}^{2}$ by a general product $M \times M$ or $M \times N$ can provide tremendous additional difficulties. Again, under additional symmetry assumptions, also this more general situation can be settled (see Theorem 3.3). As the different citations indicate, also this conjecture will reappear several times in the article and will be a good test case for different techniques and additional assumptions.

In order to extend the set of examples of non-negatively curved manifolds one makes use of

Theorem 2.3 (O'Neill) Let $(M, g)$ and $(N, g)$ be two Riemannian manifolds and $f: M \rightarrow N$ a Riemannian submersion. Then the sectional curvatures are related by

$$
K_{N}(X, Y)=K_{M}(\tilde{X}, \tilde{Y})+\frac{3}{4}\left|[\tilde{X}, \tilde{Y}]^{v}\right|^{2}
$$

where $K_{N}$ and $K_{M}$ denote respective sectional curvatures, $X, Y$ are orthonormal vector fields on $N, \tilde{X}$ and $\tilde{Y}$ are their horizontal lifts to $M$, and $[\cdot, \cdot]^{v}$ denotes the vertical projection of the Lie bracket to the fibre of the submersion.

In particular, the sectional curvature does not decrease under the projection.

One may apply this theorem, for example, to compact homogeneous spaces, i.e. to the submersion

$$
H \hookrightarrow G \rightarrow G / H
$$


where the compact Lie group $G$ is equipped with a biinvariant metric, $H \subseteq G$ is a closed subgroup, and $G / H$ is given the submersion metric. This yields non-negative curvature on $G / H$.

The same argument extends to biquotients: Let $G$ be a compact connected Lie group and let $H \subseteq G \times G$ be a closed (Lie) subgroup.

Then $H$ acts on $G$ on the left by $\left(h_{1}, h_{2}\right) \cdot g=h_{1} g h_{2}^{-1}$. The orbit space $G / H$ of this action is called the biquotient $G / / H$ of $G$ by $H$. If the action of $H$ on $G$ is free, then $G / / H$ possesses a manifold structure. This is the only case we shall consider.

If $H$ has the form $H_{1} \times H_{2}$ with $H_{1} \subseteq G \times\{1\}$ and $H_{2} \subseteq\{1\} \times G$, we also write $H_{1} \backslash G / H_{2}$ for $G / / H_{1} \times H_{2}$.

Clearly, the category of biquotients contains the one of homogeneous spaces. For this we assume that $H$ is a subgroup not only of $G \times G$, but of $G \times\{1\}$. So $H$ acts freely by left-multiplication on $G$ and the quotient is the homogeneous space $G / H$.

It was shown in [20] that a biquotient $G / / H$ is diffeomorphic to a biquotient of $G \times G$ by $G \times H$, i.e. to $\Delta G \backslash G \times G / H$ where $\Delta G$ denotes the diagonal inclusion. Thus both approaches end up to be equivalent for us. (As we can choose more complicated metrics on $G \times G$ this gives actually rise to more interesting examples of non-negatively curved spaces by O'Neill.)

Another interesting construction is due to Cheeger: We consider so-called (simplyconnected) compact rank one symmetric spaces, namely

$$
\mathbb{S}^{n}, \mathbb{C} \mathbf{P}^{n}, \mathbb{H} \mathbf{P}^{n}, \mathrm{CaP}^{2}
$$

They all admit metrics of positive curvature. Note, in particular, that we have the Hopf fibrations

$$
\begin{aligned}
& \mathbb{S}^{1} \hookrightarrow \mathbb{S}^{2 n+1} \rightarrow \mathbb{C} \mathbf{P}^{n} \\
& \mathbb{S}^{3} \hookrightarrow \mathbb{S}^{4 n+3} \rightarrow \mathbb{H} \mathbf{P}^{n}
\end{aligned}
$$

respectively a twistor fibration

$$
\mathbb{S}^{2} \hookrightarrow \mathbb{C} \mathbf{P}^{2 n+1} \rightarrow \mathbb{H} \mathbf{P}^{n}
$$

producing positive curvature on $\mathbb{C} \mathbf{P}^{n}$ and $\mathbb{H} \mathbf{P}^{n}$ from positive curvature on $\mathbb{S}^{n}$ due to O’Neill.

We have already discussed product metrics above. It is natural to ask for "sums", consequently. Let us avoid the slightly technical definition of the concept of a connected sum of two manifolds, which is the adequate notion to consider at this place. Instead, let us illustrate this concept by a sequence of pictures reflecting the idea that the connected sum of two manifolds consists of "cutting out small disks" on each one and of suitably "gluing them" along the boundary spheres (see Fig. 2).

This then naturally prompts the question: given two-negatively curved Riemannian manifolds $\left(M, g_{1}\right)$ and $\left(N, g_{2}\right)$, can we construct a metric of non-negative curvature on the connected sum $M \# N$ ? Our intuition, trained by Fig. 1, and applied to the "gluing region" actually makes this hard to believe. Indeed, in general, this should not hold true due to the topological conjectures we shall discuss later; and, as we shall see, there are some cases were it can be proven that this is actually impossible. 
Fig. 2 The connected sum of two tori
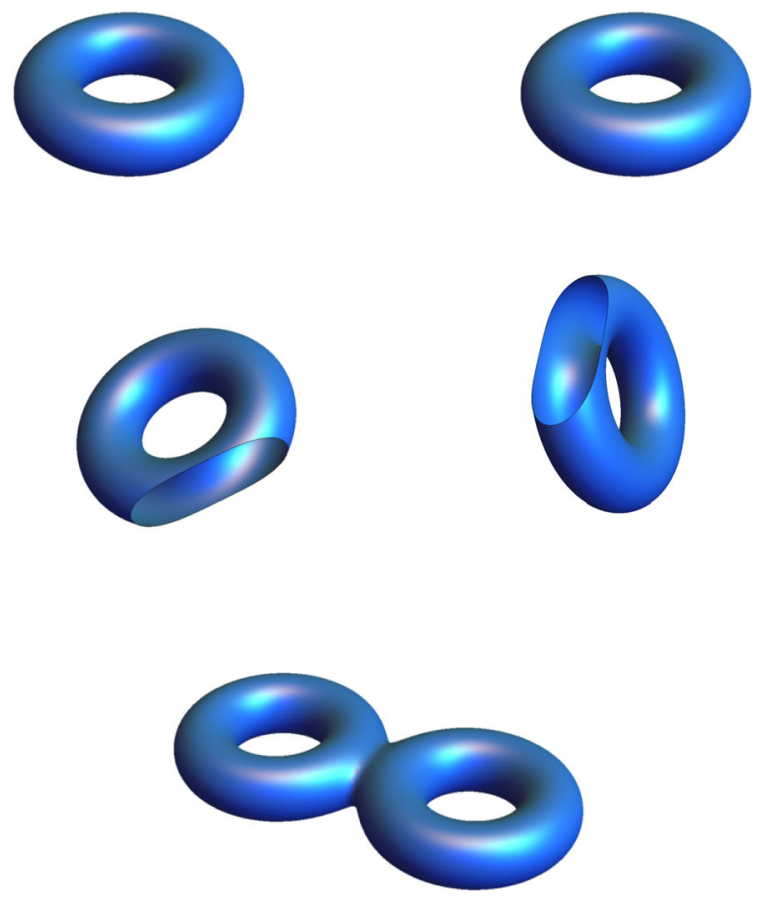

For an elaborate discussion of this see Remark 2.16 and the arguments preceding it. However, in a very special case, such non-negative curvature does not contradict the conjectures and actually can be constructed.

Theorem 2.4 (Cheeger [17]) The connected sum of a compact symmetric space of rank one with another compact symmetric space of rank one or its negative admits a metric of non-negative curvature. tion.

By the "negative", for example $\overline{\mathbb{C} \mathbf{P}^{n}}$, we refer to the space with opposite orienta-

It was shown in [61] that several of these Cheeger manifolds actually possess the structure of a biquotient like $\mathbb{C} \mathbf{P}^{n} \# \overline{\mathbb{C} \mathbf{P}^{n}}, \mathbb{C} \mathbf{P}^{n} \# \mathbb{C} \mathbf{P}^{n}, \mathbb{H} \mathbf{P}^{n} \# \overline{\mathbb{H} \mathbf{P}^{n}}, \mathrm{CaP}^{2} \# \overline{\mathrm{CaP}^{2}}$, or $\mathbb{C P}^{2 n} \# \overline{\mathbb{H} \mathbf{P}^{n}}$, whereas, for example, neither $\mathrm{CaP}^{2} \# \mathrm{CaP}^{2}$ nor $\mathbb{C P}^{4 n} \# \mathbb{H} \mathbf{P}^{2 n}$ are homotopy equivalent to a biquotient. This improves Cheeger's construction, which uses a smooth deformation to obtain non-negative curvature, by endowing the spaces with real analytic non-negatively curved metrics.

Example 2.5 The manifold $\mathbb{C} \mathbf{P}^{n} \# \overline{\mathbb{C} \mathbf{P}^{n}}$ is an $\mathbb{S}^{2}$-bundle over $\mathbb{C} \mathbf{P}^{n-1}$.

Indeed, it can be written in the form $\left(\mathbb{S}^{2 n-1} \times \mathbb{S}^{2}\right) / \mathbb{S}^{1}$ where $\mathbb{S}^{1}$ acts freely on $\mathbb{S}^{2 n-1}$ and by rotations on $\mathbb{S}^{2}$. This way, we use the principal $\mathbf{U}(1)$-bundle

$$
\mathbf{U}(1) \hookrightarrow \mathbf{S U}(n) / \mathbf{S U}(n-1) \rightarrow \mathbf{S U}(n) / \mathbf{S}(\mathbf{U}(n-1) \times \mathbf{U}(1))
$$


with total space $\mathbb{S}^{2 n+1}$, base $\mathbb{C} \mathbf{P}^{n}$, and fibre $\mathbb{S}^{1} \cong \mathbf{U}(1)$ in order to associate the onepoint completion of the standard representation of $\mathbb{S}^{1}$ on $\mathbb{R}^{2}$ resulting in the depicted $\mathbb{S}^{2}$-bundle over $\mathbb{C} \mathbf{P}^{n-1}$.

The fact that we may write $\mathbb{C} \mathbf{P}^{n} \# \overline{\mathbb{C} \mathbf{P}^{n}}$ in this form is due to the following reasoning. Removing an $\mathbb{S}^{1}$-equivariant open disc from $\mathbb{C} \mathbf{P}^{n}$ leaves us with the closed tubular neighbourhood of $\mathbb{C} \mathbf{P}^{n-1}$, i.e. the closed $\mathbb{S}^{1}$-homogeneous 2-disc bundle over $\mathbb{C} \mathbf{P}^{n-1}$. We glue two of these along the common boundary $\mathbb{S}^{1}$-bundle leaving us with an $\mathbb{S}^{2}$-bundle (in which the boundary $\mathbb{S}^{1}$ becomes the equator), namely exactly the one described above.

We may write the bundle above as a biquotient of the form

$$
\begin{aligned}
& \mathbf{U}(1) \backslash(\mathbf{S U}(n-1) / \mathbf{S U}(n-2) \times \mathbf{S O}(3) / \mathbf{S O}(2)) \\
= & \mathbf{U}(1) \backslash \mathbf{S U}(n-1) \times \mathbf{S O}(3) /(\mathbf{S U}(n-2) \times \mathbf{S O}(2))
\end{aligned}
$$

Recall that there are 28 oriented differentiable structures on the 7-dimensional "homeomomorphism spheres"- that is, on those manifolds homeomorphic to $\mathbb{S}^{7}$ yielding so-called exotic spheres, i.e. manifolds homeomorphic but not diffeomorphic to the standard sphere. It was shown by Milnor that 16 of them, the Milnor spheres, are obtainable as $\mathbb{S}^{3}$-bundles over $\mathbb{S}^{4}$. Neglecting orientation, there are 15 diffeomorphism types, 11 occurring as Milnor spheres. By work of Gromoll-Meyer, GroveZiller it was shown that the Milnor spheres admit metrics of non-negative curvature. It was recently proved that

Theorem 2.6 (Goette-Kerin-Shankar, [29]) All exotic 7-spheres admit an SO(3)invariant Riemannian metric of non-negative curvature.

The so-called Gromoll-Meyer sphere is the only 7-sphere $\mathbf{S p}(2) / / \mathbf{S p}(1)$ which can be written as a biquotient. This comes out of a more general classification (see [46], [61]) of biquotients the rational cohomology algebra of which is generated by one element only. The (free) biquotient action in this case is given by

$$
q \cdot Q:=\left(\begin{array}{ll}
q & 0 \\
0 & q
\end{array}\right) Q\left(\begin{array}{ll}
\bar{q} & 0 \\
0 & 1
\end{array}\right)
$$

for $q \in \mathbf{S p}(1), Q \in \mathbf{S p}(2)$.

Note further that vastly generalising the gluing constructions above there are several examples of non-negatively curved cohomogeneity one manifolds, i.e. manifolds $M$ admitting a $G$-action with 1 -dimensional orbit space. A principal orbit of the action has codimension 1 . Since the manifold is compact, the orbit space is either an $\mathbb{S}^{1}$ or a closed interval. In the latter case it may be shown that the cohomogeneity 1 manifold has the structure of two mapping cylinders which are glued along a principal orbit. As an example we consider the standard rotation of $\mathbb{S}^{1}$ on $\mathbb{S}^{2}$ (see Figure 3), which has an orbit space given by $[-1,1]$. At \pm 1 an orbit is singular; it actually just equals a fixed point. Over each point in $(-1,1)$ of the orbit space, the orbit is principal and equals $\mathbb{S}^{1}$. The sphere $\mathbb{S}^{2}$ now results from gluing the two mapping cylinders $\left([0,1] \times \mathbb{S}^{1}\right) /\left(\{1\} \times \mathbb{S}^{1}\right)$ and $\left([0,-1] \times \mathbb{S}^{1}\right) /\left(\{-1\} \times \mathbb{S}^{1}\right)$ via the identity at $\{0\} \times \mathbb{S}^{1}$. 
Fig. 3 A cohomogeneity 1 structure on the sphere

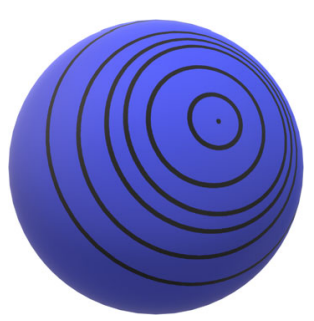

Theorem 2.7 (Grove-Ziller) A compact cohomogeneity one G-manifold has a $G$ invariant metric with non-negative sectional curvature if the codimensions of the two non-principal orbits are at most 2 each.

Theorem 2.8 (Verdiani) An even dimensional simply-connected cohomogeneity one manifold with an invariant metric of positive sectional curvature is equivariantly diffeomorphic to a compact rank one symmetric space with a linear action.

\subsubsection{Positive Curvature}

There are far less (simply-connected) examples of positively curved manifolds known than in non-negative curvature. Beside the compact rank one symmetric spaces there are the homogeneous flag manifolds due to Wallach,

$$
W^{6}=\mathbf{S U}(3) / T^{2}, \quad W^{12}=\mathbf{S p}(3) / \mathbf{S p}(1)^{3}, \quad W^{24}=\mathbf{F}_{4} / \mathbf{S p i n}(8),
$$

the Berger space

$$
B^{13}=\mathbf{S U}(5) / \mathbf{S p}(2) \cdot \mathbb{S}^{1},
$$

the Aloff-Wallach spaces

$$
W_{p, q}^{7}=\mathbf{S U}(3) / \operatorname{diag}\left(z^{p}, z^{q}, \bar{z}^{p+q}\right)
$$

with $\operatorname{gcd}(p, q)=1$ and with $p \geq q \geq 0$ and $(p, q) \neq(1,0)$ as well as the Berger space

$$
B^{7}=\mathbf{S O}(5) / \mathbf{S O}(3)
$$

for the inclusion in the latter case as well as an elaborate discussion of all of the mentioned examples we refer the reader to [67].

Beside the homogeneous examples, there are related constructions leading to positively curved biquotients. There is an analogue of the 6-dimensional flag manifold, namely

$$
\mathbf{S U}(3) / / T^{2}
$$

Then, in dimension 7, there exists the family of Eschenburg spaces

$$
\mathbf{S U}(3) / / \mathbb{S}^{1}
$$


(parametrised over different rotation numbers of the $\mathbb{S}^{1}$ ) generalising the AloffWallach spaces. The 6-dimensional space $\mathbf{S U}(3) / / T^{2}$ can actually be considered as an $\mathbb{S}^{1}$-quotient of the Eschenburg spaces which endows it with positive curvature by O'Neill. This situation in dimension 7 parallels a similar phenomenon in dimension 13, where the Bazaikin space

$$
\mathbf{S U}(5) / /\left(\mathbf{S p}(2) \cdot \mathbb{S}^{1}\right)
$$

generalise the Berger space. (For more details and the respective representations of the denominator groups see [66]). Recently, cohomogeneity one constructions in dimension 7 led to two more examples-[19, 39, 55]

\subsection{Obstructions}

Recall that by Bonnet-Myers the fundamental group of $M$ is finite under positive Ricci curvature, by Synge it is either 0 or $\mathbb{Z}_{2}$ in the even-dimensional positively curved case. (Note that this readily shows that $\mathbb{R} \mathbf{P}^{2} \times \mathbb{R} \mathbf{P}^{2}$ does not carry a metric of positive sectional curvature, whereas we have seen that the corresponding question for its finite cover $\mathbb{S}^{2} \times \mathbb{S}^{2}$ is completely open. This nicely illustrates that the simply-connected case is more cumbersome.) Work of Kapovitch-PetruninTuschmann ([47]), Kapovitch-Wilking ([45]) and Breuillard-Green-Tao ([15]) sheds more light on the structure of the fundamental group of a manifold with non-negative Ricci curvature. In particular, the group is virtually nilpotent.

Due to this, we focus on the simply-connected case (for closed manifolds) in which there are just very few obstructions known; and the ones known are not very strong. Since positive sectional curvature implies positive scalar curvature, we have seen that the $\alpha$-invariant of a closed simply-connected positively curved spin manifold vanishes. Up to a deformation argument we may, without restriction, actually see that the vanishing of the $\alpha$-invariant indeed even obstructs the existence of a nonnegatively curved metric on a closed manifold.

Gromov's Betti number bound states that on a compact non-negatively curved manifold $M^{n}$ there exists a constant $c(n)$-only depending on $n=\operatorname{dim} M$ - which bounds all $b_{i}\left(M^{n} ; \mathbb{k}\right) \leq c(n)$ with arbitrary coefficient field $\mathbb{k}$. The number $c(n)$ is explicit, yet very large even after improvements made by Abresch. In particular, this clearly yields an upper bound-yet usually tremendously large-for the Euler characteristic of $M$. Nonetheless, this upper bound lets us draw the following conclusion.

Example 2.9 Let $M$ be a closed simply-connected manifold of dimension at least 4, which is not homeomorphic to a sphere. The confirmation of the Poincaré conjecture yields that since $M$ is not homeomorphic to a sphere it cannot be homotopy equivalent to one. Hence in some coefficient field $\mathbb{k}$ the manifold $M$ does have a non-trivial Betti number $b_{j}=\operatorname{dim} H^{*}(M ; \mathbb{k}) \geq 1$ with $1<j<\operatorname{dim} M$. A Meyer-Vietoris argument readily implies that Betti numbers (different from those in degree 0 or $\operatorname{dim} M$ ) are additive under connected sums. In particular, we derive that

$$
b_{j}(M \# \stackrel{(k)}{.} \# M)=k \cdot b_{j} \geq k
$$


It follows that for $k>c(n)$ the connected sum $M \# \stackrel{(k)}{.}$ \# $M$ cannot carry a metric of non-negative sectional curvature. 回

Note that the general question when a connected sum or a product may carry non-negative or positive curvature remains open. However, clearly, any product of non-negatively curved metrics will be non-negatively curved.

These are the general known bounds to non-negative and at the same time positive sectional curvature. However, the Gauss-Bonnet theorem in dimension 2 lets us relate the Euler characteristic of a closed oriented surface to sectional curvature.

$$
\int_{M} K \mathrm{dvol}=2 \pi \chi(M)
$$

Thus, the Euler characteristic is non-negative if and only if $M$ carries a metric of nonnegative curvature, it is positive if and only if $M$ is positively curved, i.e. $\mathbb{S}^{2}$ or $\mathbb{R} \mathbf{P}^{2}$. Since the Euler characteristic of an orientable closed surface is related to its genus by $\chi(S)=2-2 g$, we see that no such surface of genus $g \geq 2$ will admit a metric of non-negative curvature.

The situation in dimension 2 motivates some general conjectures.

\subsection{Conjectures}

We list several conjectures which connect non-negative and positive curvature to topology. Some of these are highly interdependent respectively follow from another.

Let us begin with conjectures involving cohomology. We have already seen one "Hopf conjecture", there is a second one different in nature speculating about similar implications as we drew them from the Gauss-Bonnet theorem in dimension 2. Clearly, by Poincaré duality the Euler characteristic of a simply-connected closed odd-dimensional manifold vanishes.

Conjecture 2.10 (Hopf) Let $M^{2 n}$ be a closed manifold, then

$$
0 \quad \begin{cases}\leq(-1)^{n} \chi(M) & \text { if } M \text { is non-positively curved } \\ \leq \chi(M) & \text { if } M \text { is non-negatively curved } \\ <\chi(M) & \text { if } M \text { is positively curved }\end{cases}
$$

The following upper bound was conjectured by Gromov when establishing his bound on Betti numbers.

Conjecture (Gromov) A non-negatively curved manifold $M^{n}$ satisfies $b_{k}(M) \leq\left(\begin{array}{l}n \\ k\end{array}\right)$.

By $T^{n}=\mathbb{S}^{1} \times \stackrel{(n)}{.} \times \mathbb{S}^{1}$ we denote the $n$-torus. The Künneth formula lets us easily derive that $b_{k}\left(T^{n}\right)=\left(\begin{array}{l}n \\ k\end{array}\right)$. Moreover, the torus admits a flat metric. Hence, the conjecture speculates that a general non-negatively curved space has topology bounded by the one of the torus. 
Obvious consequences of this conjecture are that

$$
\sum_{k} b_{k}(M) \leq \sum_{k} \operatorname{rk} H^{k}\left(T^{n}\right)=2^{n}
$$

and, in particular, that

$$
\chi(M) \leq 2^{n}
$$

Certainly the strongest and most far-reaching conjecture in this context is the following. Recall that the homotopy groups $\pi_{i}(M)$ of a topological space $M$ are abelian for $i \geq 2$. By $\pi_{*}(M)$ we denote $\bigoplus_{i} \pi_{i}(M)$. A simply-connected (or, more generally, nilpotent, i.e. the fundamental group is nilpotent acting nilpotently on higher homotopy groups) topological space is called rationally elliptic if both its rational cohomology algebra and its total rational homotopy $\pi_{*}(M) \otimes \mathbb{Q}$ are finite dimensional. Clearly, on a closed manifold $M$ this boils down to the question whether from some degree on all the homotopy groups are torsion or not.

Conjecture (Bott-Grove-Halperin) It holds that $\operatorname{dim} \pi_{*}(M) \otimes \mathbb{Q}<\infty$, i.e. $M$ is rationally elliptic, if $M$ is non-negatively curved.

Remark 2.11 Rational ellipticity may be reformulated into a polynomial growth condition on rational loop space cohomology. This condition then may be considered with arbitrary field coefficients giving rise to a general notion of ellipticity postulating this polynomial growth irrespective of coefficients.

Recall that a closed smooth manifold $M$ is called almost non-negatively curved, if it admits a sequence of Riemannian metrics $\left\{g_{n}\right\}_{n \in \mathbb{N}}$ such that its sectional curvatures satisfy $K\left(M, g_{n}\right) \geq-1 / n$ and such that the diameters can be restricted by $\operatorname{diam}\left(M, g_{n}\right) \leq 1 / n$.

We may combine this to the most general form of this conjecture, which probably should be that an almost non-negatively curved manifold is elliptic. 回

Let us bring another classical property from rational homotopy into play: Recall that a topological space is called formal if its rational homotopy type is fully determined by its rational cohomology algebra. On smooth manifolds this is equivalent to the existence of a chain of quasi-isomorphisms (morphisms inducing isomorphisms in cohomology) from the commutative differential graded algebra of smooth differential forms $\Omega(M)$ to the cohomology algebra (equipped with the zero-differential):

$$
\Omega(M) \stackrel{\simeq}{\rightarrow} \ldots \stackrel{\simeq}{\leftarrow} \ldots \stackrel{\simeq}{\rightarrow}\left(H^{*}(M ; \mathbb{R}), 0\right)
$$

(Clearly, we need to consider these chains, since any such quasi-isomorphism need not be invertible.) It is clear that such a weak equivalence with rational coefficients implies one with real coefficients. Although the real homotopy type does not determine the rational homotopy type in general, it is an astounding observation by Halperin-Stasheff that formality indeed does not depend on the extension field of $\mathbb{Q}$.

In particular, formality implies the vanishing of Massey products. Formality is known for several examples (like compact Kähler manifolds, symmetric spaces or 
compact homogeneous spaces $G / H$ with $\operatorname{rk} G=\operatorname{rk} H$ ) and is conjectured not only in special holonomy, but also in the following context.

Conjecture 2.12 A simply-connected closed manifold of positive curvature is a formal space.

Contrast this conjecture to results on "geometric formality" in the context of positive curvature. This will be discussed in Sect. 3.2.

Remark 2.13 Let us highlight some interdependencies of all these conjectures.

- Both the Hopf conjecture for non-negative curvature and the conjecture by Gromov would directly follow from the Bott-Grove-Halperin conjecture. Indeed, the structure theory of rationally elliptic spaces yields that they always have non-negative Euler characteristic. Furthermore, the Euler characteristic is positive if and only if odd-degree Betti numbers vanish. For biquotients $G / / H$ this is equivalent to rk $G=\operatorname{rk} H$.

Moreover, rationally elliptic spaces satisfy Poincaré duality and their Betti numbers are bounded from above by the ones of the torus of the same dimension.

- The formality conjecture for positive curvature would follow from the Hopf conjecture (in positive curvature) and the Bott-Grove-Halperin conjecture. Indeed, as depicted, in this case we are dealing with a rationally elliptic space of positive Euler characteristic. These spaces are known to be formal, in particular.

- Formality does not necessarily hold in non-negative curvature. This follows, for example, from the existence of non-formal homogeneous spaces like $\mathbf{S p}$ (5)/SU(5) (cf. [1]). Recall that a biinvariant metric on $\mathbf{S p}(5)$ (which is non-negatively curved) together with O'Neill's theorem yield non-negative curvature on the quotient.

- The formality conjecture has no implications on the other two ones (Hopf and Bott-Grove-Halperin), since there are hyperbolic (i.e. non-elliptic) formal manifolds (like multi-summand connected sums) which may at the same time have negative Euler characteristic whilst being formal-as, for example, $T^{2} \# T^{2}$. More precisely, the connected sum of two formal manifolds is again formal. As we have seen, Betti numbers are additive under connected sums (except for the one in degree zero and the one in top degree). It follows that $b_{1}\left(T^{2} \# T^{2}\right)=4$ and $\chi\left(T^{2} \# T^{2}\right)=-2$. In particular, this manifold is not rationally elliptic. 回

Example 2.14 Compact Lie groups are rationally elliptic, as a theorem by Hopf on $\mathrm{H}$-spaces (spaces with a "multilpication" and an "identity element") asserts. Indeed, their rational cohomology is given by a graded exterior algebra. This implies that their rational homotopy type is encoded by the very same algebra together with a trivial differential.

Next the long exact sequence of homotopy groups yields that homogeneous spaces and, more generally, biquotients are rationally elliptic. So are cohomogeneity one manifolds (see [35]). This amounts to the fact that all known examples of nonnegative curvature are rationally elliptic. A direct check shows that they are all formal of non-negative Euler characteristic (respectively positive Euler characteristic for positive curvature), as well. 回 
We can further connect these conjectures to Cheeger's theorem 2.4 above. First recall from Remark 2.13 that the connected sum of two (nilpotent) formal topological spaces is formal (the same is true for the direct product). The Euler characteristic of the connected sum of two compact rank one symmetric spaces is non-negative, which, however, is no longer true for $\chi\left(\mathbb{R} \mathbf{P}^{4} \# \mathbb{R} \mathbf{P}^{4} \# \mathbb{R} \mathbf{P}^{4}\right)=-1$.

Proposition 2.15 A connected sum $X=X_{1} \#$ (k). \# $X_{k}$ of $k$ simply-connected compact rank one symmetric spaces-each different from the sphere-(irrespective of their orientation) is rationally elliptic if and only if $k \leq 2$.

Proof The fundamental group of the connected sum $M \# N$ of two manifolds $M, N$ is the free product $\pi_{1}(M) * \pi_{1}(N)$. Hence, in our case, the connected sum $X$ stays simply-connected.

Although we try to make the arguments as self-explanatory as possible, the subsequent reasoning relies on some basic knowledge of rational homotopy theory as can be found in [23]. By a Mayer-Vietoris argument Betti numbers of connected sums are additive in positive degrees below the dimension. In particular, the Euler characteristic of $X$ is positive. Thus if $X$ was elliptic, it would be a pure space (see $\left[23\right.$, p. 435]) with $\operatorname{dim} \pi_{\text {even }}(X) \otimes \mathbb{Q}=\operatorname{dim} \pi_{\text {odd }}(X) \otimes \mathbb{Q}$. Hence each even-degree rational homotopy group consists of spherical cohomology, i.e. cohomology in the image of the Hurewicz morphism. In other words, since the collapse maps $X \rightarrow X_{i}$ induce cohomology inclusions by Mayer-Vietoris, the cohomology algebra of $X$ is generated by $k$ elements $x_{1}, \ldots, x_{k}$ in even degrees-each $x_{i}$ generating $H^{*}\left(X_{i} ; \mathbb{Q}\right)$ as an algebra. These $x_{i}$ hence completely generate even-degree rational homotopy, i.e. $\operatorname{dim} \pi_{\text {even }}(X)=k$. Moreover, we see that $x_{i} x_{j}=0$ in $H^{*}(X ; \mathbb{Q})$ if $i \neq j$. This implies that there are $\left(\begin{array}{l}k \\ 2\end{array}\right)$ independent relations which are realised by as many generators of $\pi_{\text {odd }}(X) \otimes \mathbb{Q}$. Consequently, $\left(\begin{array}{l}k \\ 2\end{array}\right)$ may not exceed $\operatorname{dim} \pi_{\text {even }}(X)=k$. It follows that $k \leq 3$. If $k=3$, the relations $x_{i} x_{j}=0$ do not cover that also suitable powers of the $x_{i}$ are glued to yield a common fundamental form of $X$. This leads to the existence of another odd-degree rational homotopy group. It follows that $k \in\{1,2\}$.

If $k=2$, it holds that $H^{*}(X ; \mathbb{Q}) \cong \mathbb{Q}\left[x_{1}, x_{2}\right] /\left(x_{1}^{l_{1}} \pm x_{2}^{l_{2}}, x_{1} x_{2}\right)$ (for some $l_{1}$, $l_{2} \geq 2$ ). Hence it is positively elliptic, i.e. rationally elliptic with positive Euler characteristic.

Remark 2.16 Let us summarise the situation for connected sums using the example of $\mathbb{C} \mathbf{P}^{2}$ and its multiples.

- $\mathbb{C} \mathbf{P}^{2}$ carries a metric of positive curvature (by O'Neill).

- $\mathbb{C} \mathbf{P}^{2} \# \mathbb{C} \mathbf{P}^{2}$ admits a metric of non-negative curvature by Cheeger (see Theorem 2.4).

- The connected sum $\mathbb{C} \mathbf{P}^{2} \# . .(k)$. \# $\mathbb{C} \mathbf{P}^{2}$ for $k \geq 3$ is not rationally elliptic (see Proposition 2.15), and hence should not carry non-negative curvature.

- The 4-dimensional connected sum $\mathbb{C} \mathbf{P}^{2} \# . .(k)$ \# $\mathbb{C} \mathbf{P}^{2}$ for $k \geq 3$ has second Betti number $k$. The corresponding Betti number of the 4-dimensional torus is $\left(\begin{array}{l}4 \\ 2\end{array}\right)=6$. Hence Gromov's conjecture is infringed for $k \geq 7$. 
- Due to Gromov's Betti number bound-which only depends on the dimension of the manifold, that is 4 in our case-we hence derive that there is some $k$ such that $\mathbb{C} \mathbf{P}^{2} \# \stackrel{(k)}{.} \# \mathbb{C} \mathbf{P}^{2}$ does not admit a metric of non-negative curvature. (Note that such a reasoning can be used to construct manifolds of positive Ricci curvature but lacking any metric of non-negative sectional curvature.)

- The Euler characteristic of $\mathbb{C} \mathbf{P}^{2} \# .(k) . \# \mathbb{C} \mathbf{P}^{2}$ is positive, and the connected sum of formal spaces is formal-clearly, $\mathbb{C} \mathbf{P}^{2}$ is formal. 回

One may be tempted to extend the Hopf conjecture on products to connected sums in the following way.

Question 2.17 Let $M$ and $N$ be simply-connected closed manifolds such that none of them (rationally) is a sphere. When does M\#N admit a metric of non-negative or of positive curvature?

(Recall that the connected sum with a sphere does not change the diffeomorphism type of a manifold.) Again see [5] for results on this in the class of metrics invariant under torus actions.

Let us finally present a conjecture which comes from differential topology, more precisely from index theory. We refer the reader to [43] for an introduction to the latter. Here we are interested in how certain expressions in characteristic classes evaluate at the fundamental class of the manifold, i.e. when being integrated over the manifold. The values of certain such characteristic numbers then often can be related to indices of elliptic differential operators.

For us one important such combination characteristic of numbers is given by the elliptic genus $\Phi(X)$. Recall its definition via

$$
\Phi(X)=q^{-k / 2} \cdot \hat{A}\left(X, \prod_{n \geq 1, \text { odd }}^{\infty} \bigwedge_{-q^{n}} T_{\mathbb{C}} \cdot \prod_{n \geq 2, \text { even }}^{\infty} S_{q^{n}} T_{\mathbb{C}}\right)
$$

on an oriented connected compact manifold $X^{n}$ with $n \equiv 0 \bmod 4\left(\right.$ where $T_{\mathbb{C}}$ denotes the complexified tangent bundle of $X$ and $\bigwedge_{t} T_{\mathbb{C}}=\sum_{k=0}^{\infty}\left(\bigwedge^{k} T_{\mathbb{C}}\right) \cdot t^{k}$ is a formal power series over exterior powers and $S_{t} T_{\mathbb{C}}=\sum_{k=0}^{\infty}\left(S^{k} T_{\mathbb{C}}\right) \cdot t^{k}$ one over symmetric powers of $T_{\mathbb{C}}$. The elliptic genus is a Laurent series in the variable $q$ with coefficients $\hat{A}(X), \hat{A}\left(X, T_{\mathbb{C}} X\right), \hat{A}\left(X, \bigwedge^{2} T_{\mathbb{C}} X\right)$, etc.

As we have seen the $\hat{A}$-genus vanishes under the existence of a metric of positive scalar curvature on spin manifolds (where it corresponds to the index of the Dirac operator, and the result follows from Lichnerowicz' formula). In particular, under positive sectional curvature, the first non-trivial coefficient of the elliptic genus vanishes. The following conjecture speculates that actually all such coefficients, i.e. all of these twisted $\hat{A}$-genera should vanish under the existence of positive curvature leaving only a constant term.

Conjecture 2.18 (Dessai) Let $(M, g)$ be a spin manifold admitting a metric of positive curvature. Then the elliptic genus is a constant power series. 
Compare this to [42] where it is proved that the elliptic genus is constant on $4 k$ dimensional spin homogeneous spaces. Expressing the elliptic genus as a power series of twisted signatures, it can be seen that in the latter case it equals the (usual) signature.

Let us finally present another conjecture which cannot be generalised from positive curvature to non-negative curvature.

Conjecture (Petersen-Wilhelm) Let $M \rightarrow B$ be a (non-trivial) Riemannian submersion with $M$ a complete positively curved manifold, then $2 \operatorname{dim} B>\operatorname{dim} M$.

We may suppose all positively curved manifolds to be compact. If $M$ is not compact, Walschap proved that there are no non-trivial submersions from $M$ (see [63, Theorem 2.1]). Then the submersion is a fibre bundle (with fiber $F$ ) according to Ehresmann and the conjecture can be stated as $\operatorname{dim} B>\operatorname{dim} F$.

The conjecture was confirmed for the known simply-connected even-dimensional examples of positive curvature in [4] and for the known simply-connected odddimensional examples in [31]. It seems that this conjecture is very geometrical in nature lacking the connection to topology on which we focus in this survey. However, the proofs confirming the conjecture on the known examples draw essentially first on the structure of rational fibrations and second on integral computations in the Leray-Serre spectral sequence.

As announced this conjecture is again a "specialty" of positive curvature and is easily seen to fail in non-negative curvature. It suffices to consider the product metric on $M \times N$-both factors non-negatively curved and $\operatorname{dim} M<\operatorname{dim} N$-and the projection bundle $N \hookrightarrow M \times N \rightarrow M$.

\section{Positive Curvature and...}

Due to the lack of examples and general techniques the study of positively curved metrics seems rather complicated. Therefore one often draws on auxiliary assumptions. We shall present two of them: isometric group (mainly torus) actions and geometric formality.

\section{1 ...Symmetry}

Especially for this section we refer the reader to the much more elaborate corresponding section of [2]. As both examples and coincidences in the respective theories show, isometric group actions behave very well with positive curvature. In particular, representation and Lie theory then are extremely helpful. As for isometric torus actions there is another proof scheme which enters the stage: Since positive curvature (by the Weinstein fixed-point theorem) grants the existence of torus fixed-points in even dimensions, and since fixed-point components are totally geodesic, one may work inductively over (the dimension of) the fixed-point components. That is, a fixed-point component is again positively curved, and one would like to apply an induction assumption to it. The induction assumption usually is formulated connecting the rank 
of an isometrically acting torus to the dimension of the manifold. Hence, in order to be able to apply an induction assumption to such a fixed-point component, it remains to see that it is not fixed by too large a torus. In other words, one has to make sure that a certain, large enough subtorus, of the torus acting on the ambient manifold indeed acts effectively on the fixed-point component.

The basic proof scheme is as follows: Either the codimension of a fixed point component is "small" (such that the inductive assumption on the rank of the torus acting effectively cannot be maintained), or the codimension of the entire fixed-point set is "large" in the sense that the induction assumption may be applied to any of its components. In the first case one always needs a different argument to conclude something on the structure of the manifold.

Examples of such "inductive results" are the following: The classification of positively curved manifolds $M^{n}$ admitting an effective isometric action of a torus $T^{\lfloor(n+1) / 2\rfloor}$ respectively $T^{\lfloor(n-1) / 2\rfloor}$ by Grove-Searle (see [36]) respectively FangRong (see [22]), homotopy and cohomology classification results by Wilking under effective isometric $T^{n / 4+1}$ and $T^{n / 6+1}$ actions (see [65]), as well as Euler characteristic computations by Amann-Kennard under isometric torus actions-here the rank of the torus is either linear or logarithmic in the dimension of the manifold (see [3], [6]).

Exactly the same proof pattern can be applied to index theory. That is, due to a theorem by Hirzebruch-Slodowy (see [42, Corollary, p. 317]) one may apply again this inductive approach over fixed-point components.

Theorem 3.1 (Weisskopf, [64]) Let $\left(M^{n}, g\right)$ be a closed, connected, positively curved spin manifold. Suppose that a torus $T^{s}$ acts isometrically and effectively on $M$ with $n \geq 2^{s}$ and $s \geq 3$. Then, one of the following holds:

- the first $\min \left\{\lfloor n / 16\rfloor+1,2^{s-3}\right\}$ coefficients of the elliptic genus vanish, or

- the rational cohomology algebra of $M$ is 4-periodic.

Finally, as we already discussed, the Hopf conjecture on $\mathbb{S}^{2} \times \mathbb{S}^{2}$ is confirmed in the class of metrics invariant under an $\mathbb{S}^{1}$-action.

Theorem 3.2 (Hsiang-Kleiner, Grove-Wilking, [44], [37]) A simply-connected positively curved 4-manifold admitting an effective isometric $\mathbb{S}^{1}$-action is equivariantly diffeomorphic to $\mathbb{S}^{4}$ or $\mathbb{C} \mathbf{P}^{2}$.

Clearly, formulating the Hopf conjecture via metrics on $\mathbb{S}^{2} \times \mathbb{S}^{2}$, merely underlines our lack of knowledge on which metrics can appear on general products. One would extend it speculating that a fortiori on the product of less special manifolds there should not occur any positive curvature. Using symmetry the following theorem motivates this further.

Theorem 3.3 (Amann-Kennard, [5]) Let $M^{2 n}$ be a simply-connected closed manifold with $b_{4}(M)=0$. Assume $M$ admits a Riemannian manifold with positive sectional curvature invariant under the action of a torus $T$ with $\operatorname{dim}(T) \geq \log _{4 / 3}(2 n)$. Then we derive that $\chi(M)=\chi\left(\mathbb{S}^{2 n}\right)=2$. 


\section{2 ...Geometric Formality}

Recall from Sect. 2.3 that a manifold is formal if its smooth differential forms $\Omega(M)$ are connected by a chain of quasi-isomorphism to deRham cohomology $H^{*}(\Omega(M))=H^{*}(M ; \mathbb{R})$. Symmetric spaces are examples.

We consider $\mathrm{d}^{*}: \Omega^{*}(M) \rightarrow \Omega^{*-1}(M)$ and $\mathrm{d}^{*}=(-1)^{n p+n+1} * \mathrm{~d} *$ where $*$ : $\Omega^{p}(M) \rightarrow \Omega^{n-p}(M)$ is the Hodge-*-operator. The main tool and main motivation in this section is the Hodge decomposition, which shows that harmonic forms $\mathcal{H}^{*}$ represent cohomology classes. The starting point for the whole discussion of geometric formality is that this is true for the module structure of cohomology, however, harmonic forms seldom form a subalgebra of $\Omega(M)$. Let us elaborate on this, starting with the

Theorem 3.4 (Hodge decomposition) Let $(M, g)$ be a compact closed manifold. Then there is an orthogonal decomposition

$$
\Omega(M)=\mathcal{H}^{*} \oplus \operatorname{im}(\mathrm{d}) \oplus \operatorname{im}\left(\mathrm{d}^{*}\right)
$$

In particular, there is an isomorphism of graded $\mathbb{R}$-vector spaces

$$
\mathcal{H}^{*} \cong H^{*}(M ; \mathbb{R})
$$

The inclusion of the module $\mathcal{H}^{*}$ of harmonic forms hence is a quasi-isomorphism: Any non-trivial d-closed form lies in $\mathcal{H}^{*} \oplus$ imd, as a d-closed form in im $\mathrm{d}^{*}$ is also $\mathrm{d}^{*}$ closed, thus harmonic and hence equal to 0 by the direct sum decomposition. Hence, as a module, cohomology is isomorphic to harmonic forms.

In the case when harmonic forms are a subalgebra of the differential forms, the inclusion is a quasi-isomorphism of algebras and $M$ is formal. This leads to the following definition (which, however, is much stronger than formality).

Definition 3.5 A Riemannian metric is formal if wedge products of harmonic forms are again harmonic. A manifold is called geometrically formal if it admits a formal metric.

The formality of compact symmetric spaces and, indeed, their geometric formality follows from the fact that in this case harmonic forms are parallel whence their product is again parallel whence harmonic. Note that there are only very few examples of geometrically formal spaces; beside compact symmetric spaces, most prominently, one may find some geometrically formal Stiefel manifolds.

See [34, 48, 50-53] for obstructions to geometric formality and properties of such manifolds. One of these is that the sum of the Betti numbers of a geometrically formal manifold $M^{n}$ again satisfies that

$$
b_{k}\left(M^{n}\right) \leq\left(\begin{array}{l}
n \\
k
\end{array}\right)
$$

which we readily recognise as the very bound in Gromov's conjecture respectively the bound resulting from rational ellipticity. 
Let us summarise our observations: We conjectured positively curved manifolds to be formal. Moreover, this bound on Betti numbers would also be implied by the cited conjectures in positive curvature. Furthermore, at least on compact rank one symmetric spaces one may find a metric of positive curvature as well as a geometrically formal one. So it seems reasonable to try to relate geometric formality to positive curvature. This has been done in different settings.

However, for homogeneous spaces the relation between geometric formality and positive curvature is not stronger than what we already observed, i.e. we do not find new formal metrics.

Theorem 3.6 (Amann-Ziller, [9]) A homogeneous formal metric of positive curvature is either symmetric or a metric on a rational homology sphere.

In particular, even on a geometrically formal manifold, not every positively curved metric is formal-for example, this can be observed on $\mathbb{C} \mathbf{P}^{2 n+1}$ where we may scale the metric according to shrinking the fibre of the twistor fibration $\mathbb{S}^{2} \rightarrow \mathbb{C} \mathbf{P}^{2 n+1} \rightarrow$ $\mathbb{H} \mathbf{P}^{n}$.

Example 3.7 Note that any Riemannian metric on a simply-connected manifold which has the rational homotopy type or, equivalently in this case, the rational cohomology of a sphere is formal. Clearly, the only products to consider are the powers of the (harmonic) volume form. These simply vanish for degree reasons, hence are trivially harmonic. 回

Nonetheless, as mentioned before, geometric formality provides a suitable setup to confirm the Hopf conjecture on $\mathbb{S}^{2} \times \mathbb{S}^{2}$.

Theorem 3.8 (Bär, Kotschick, [11], [49]) A simply-connected geometrically formal closed oriented 4-manifold $M$ admitting a (possibly non-formal) metric of positive sectional curvature is either homeomorphic to $\mathbb{S}^{4}$, or diffeomorphic to $\mathbb{C} \mathbf{P}^{2}$.

\section{Two-sided Curvature Bounds}

Let us now focus on families of Riemannian manifolds of a fixed dimension which satisfy two-sided curvature bounds. A central question in this context is whether certain finiteness conditions within such a famlily of manifolds are fulfilled. There are various results of this type, let us just mention a few.

The Gromov Betti number estimate originally states that for universal bounds on sectional curvature and diameter, $K \geq C$, diam $\leq D$, the total Betti number, i.e. the sum over all Betti numbers, is universally bounded.

If the volume is universally bounded from below, vol $\geq v \geq 0$, curvature is bounded by $|K| \leq C$ and diameter is bounded diam $\leq D$, diffeomorphism finiteness holds, i.e. there are only finitely many diffeomorphism types of manifolds satisfying these restrictions simultaneously. Only requiring a lower curvature bound, $K \geq C$, instead of the two-sided bound in this set of prerequisites yields homeomorphism finiteness and diffeomorphism finiteness from dimension 5 on. 
In the following we denote by $K(M)$ the set of all sectional curvatures ranging over all 2-planes in the tangent bundle of $M$. In the next theorem the volume bound can be dropped, and as an additional assumption it is assumed that $M$ is $\pi_{2}$-finite, i.e. the second homotopy group $\left|\pi_{2}(M)\right|<\infty$ is torsion.

Theorem 4.1 (Petrunin-Tuschmann, [56]) For given $m, C$ and D, there is only a finite number of diffeomorphism types of simply-connected closed $m$-dimensional manifolds $M$ with finite second homotopy groups which admit Riemannian metrics with sectional curvature $|K(M)| \leq C$ and diameter $\operatorname{diam}(M) \leq D$.

More precisely, the authors show that for given $m, C$ and $D$, there is only a finite number of diffeomorphism types of simply-connected closed $m$-dimensional $\pi_{2}$-finite manifolds $E_{i}$ such that any simply-connected closed $m$-dimensional manifold $M$ admitting a Riemannian metric with sectional curvature $|K(M)| \leq C$ and diameter $\operatorname{diam}(\mathrm{M}) \leq D$ is diffeomorphic to a factor space $M=E_{i} / T^{k_{i}}$, where $0 \leq k_{i}=\operatorname{dim} E_{i}-m$ and the torus $T^{k_{i}}$ acts freely on $E_{i}$.

There is a classical finiteness theorem by Sullivan.

Theorem 4.2 (Sullivan, [60]) The diffeomorphism type of a simply-connected Kähler manifold of dimension at least 3 is determined up to finite ambiguity by its integral cohomology ring and its Pontryagin classes.

This result essentially builds on the formality of Kähler manifolds. Thus it may have also motivated

Question 4.3 (Grove) Are there only finitely many rational homotopy types of closed simply-connected manifolds with a given lower bound for the sectional curvature and a given upper bound on the diameter?

In view of the remark above, this question translates to whether it is possible to define an infinite number of inequivalent differentials on the minimal models $(\Lambda V, \mathrm{~d})$ of these manifolds.

Several negative answers to this question were found, see [21], [62], [41]—all having certain nice properties. From dimension 7 on they all have an additional upper curvature bound and they all already differ by their cohomology structure. Let us present some in more detail.

Theorem 4.4 (Totaro, [62]) There are numbers $C$ and $D$ such that there are infinitely many isomorphism classes of rational cohomology rings among simply-connected closed Riemannian 9-manifolds with curvature $0 \leq K \leq C$ and diameter bounded from above by diam $\leq D$.

Sketch of proof In view of Theorem 4.1 and its refinement which we gave below the statement of the theorem, we need to construct the examples as torus quotients of one common simply-connected rationally two-connected manifold. So we consider 9-dimensional biquotients of the form

$$
Y:=(\mathbf{S p}(1) \times \mathbf{S p}(1) \times \mathbf{S p}(1) \times \mathbf{S p}(1)) / /(\mathbf{U}(1) \times \mathbf{U}(1) \times \mathbf{U}(1))
$$


The infinitely many cohomology types will come from specifying the inclusions

$$
(\mathbf{U}(1))^{3} \hookrightarrow\left((\mathbf{U}(1))^{4}\right)^{2}=\left(T_{(\mathbf{S p}(1))^{4}}\right)^{2}
$$

i.e. the biquotient action. The fact that the denominator group has corank 1 will give us enough freedom for this.

First one specifies a biquotient action of $\mathbf{U}(1)^{4}$ in such a way that the resulting 8-dimensional quotient $M$ satisfies

$$
H^{*}(M ; \mathbb{Q})=\mathbb{Q}\left[x_{1}, x_{2}, x_{3}, x_{4}\right] /\left(x_{1}^{2}, x_{2}^{2}, x_{3}^{2}-x_{1} x_{2}, x_{4}^{2}-x_{1} x_{2}\right)
$$

It is then possible to choose a 3-parameter family of subtori $\mathbf{U}(1)^{3} \subseteq \mathbf{U}(1)^{4}$ providing respective 9-dimensional manifolds $Y$ in such a way that their rational cohomology does not fall into finitely many types. In [62] this is proven by showing that the kernel of the cup product map in degree 2 is a linear system of quadrics which even modulo coordinate changes consists of infinitely many types. The curvature properties hold due to a "close look" at O'Neill's formula.

In [41] homogeneous examples of this form are constructed in dimension 13 andby taking the direct product with a sphere with the example in dimension 13-from dimension 15 on.

For this choose

$$
B:=\left(\mathbf{S U}(3) / T^{2}\right) \times\left(\mathbf{S U}(3) / T^{2}\right)
$$

where $T^{2}$ is the respective maximal torus. Then cohomology computes as

$$
H^{*}(B ; \mathbb{Z})=\mathbb{Z}\left[x_{1}, y_{1}, x_{2}, y_{2}\right] /\left(x_{1}^{2}+x_{1} y_{1}+y_{1}^{2}, x_{1}^{2} y_{1}+x_{1} y_{1}^{2}, x_{2}^{2}+x_{2} y_{2}+y_{2}^{2}\right)
$$

In analogy to the actual procedure in the proof of Theorem 4.4 (and since $\mathbb{S}^{1}$-actions may be lifted), one may pass to the principal $\mathbb{S}^{1}$-bundle $M_{a}$ with first Chern class $x_{1}+$ $a y_{1}-y_{2}$ for integral $a$. This makes $M_{a}$ a homogeneous space of $\mathbf{S U}(3) \times \mathbf{S U}(3) \times \mathbb{S}^{1}$. The (Euler class of the) $\mathbb{S}^{1}$-factor in cohomology exactly yields the identification $x_{1}=-a y_{1}+y_{2}$. It is then shown that the resulting complex cohomology rings of the $M_{a}$ are pairwise non-isomorphic.

In the same article a family of 22-dimensional homogeneous such examples is constructed which even possess almost non-negative curvature operators.

\section{Open Manifolds of Non-negative Curvature}

While our focus was lying on closed manifolds so far, let us now consider open manifolds. Here we need to mention the famous

Theorem 5.1 (Soul theorem, Cheeger-Gromoll, [18]) Let $(N, g)$ be an open manifold with $K(N) \geq 0$, then there exists a closed submanifold $S \subseteq N$, the soul, with the properties that 
- $K(S) \geq 0$ in the induced metric,

- $S$ is totally convex,

- $N$ is diffeomorphic to the normal bundle of $S$ in $N$.

Recall that by "totally convex" we refer to the property that any geodesic between two points in $S$ completely lies in $S$ (and not only minimising ones).

The soul is not necessarily unique, but any two ones are isometric.

Example 5.2 - Any point on flat $\mathbb{R}^{n}$ is a soul.

- Only the apex on the paraboloid - which we have seen to possess non-negative curvature - is a soul. Indeed, for any other point there are geodesic loops leaving the point.

- A soul on the cylinder is a slice $\mathbb{S}^{1}$. 回

Let us see, however, that there are manifolds which possess different nonnegatively curved metrics such that the respective souls are not diffeomorphic or even not homeomorphic. For this note that there is a diffeomorphism

$$
\Sigma^{7} \times \mathbb{R}^{8} \cong \mathbb{S}^{7} \times \mathbb{R}^{8}=: N
$$

where $\Sigma^{7}$ denotes an exotic sphere. As observed above, all of them admit metrics of non-negative curvature. Consequently, providing $N$ with respective product metrics, we deduce that $N$ admits metrics with non-diffeomorphic souls. It was shown by Belegradek that the manifold $\mathbb{S}^{3} \times \mathbb{S}^{4} \times \mathbb{R}^{5}$ even possesses infinitely many pairwise non-homeomorphic souls. This was extended in

Theorem 5.3 (Belegradek-Kwasik-Schultz, [14]) For each $k \geq 3$, there are infinitely many complete metrics of non-negative sectional curvature on $N=\mathbb{S}^{4} \times \mathbb{S}^{k} \times$ $\mathbb{R}^{4}$ the souls of which are pairwise non-homeomorphic.

Another recent strengthening of this is

Theorem 5.4 (González-Zibrowius, [33]) There are simply-connected open manifolds with a pair of non-homeomorphic souls of positive sectional curvature.

We want to focus on a certain converse to the soul theorem.

Question Which vector bundles over a compact non-negatively curved manifold $S$ admit a non-negatively curved metric?

There exist examples of vector bundles over base spaces with infinite fundamental group where the total space admits no complete metric of non-negative curvature (see $[12,13,54])$. However, it seems that no obstructions are known in the case of finite fundamental group. Moreover, all real vector bundles over $\mathbb{S}^{n}$, with $2 \leq n \leq 5$, admit non-negative curvature (see [38]). For higher dimensional spheres, Rigas (see [58]) proved that for every real vector bundle $E \rightarrow \mathbb{S}^{n}, n$ arbitrary, there is some $k$ such 
that $E \times \mathbb{R}^{k}$ admits a metric of non-negative curvature. We want to think of such a statement as an existence statement "up to stabilisation" with trivial bundles.

Rigas' statement (or even stronger versions of it) were shown to hold when one replaces the base space $\mathbb{S}^{n}$ by certain classes of homogeneous spaces (see [30, 32]) or certain classes of biquotients (see [33]), etc.

In [30], [32] a stable version of the question is proved for real bundles in the case of a homogeneous positively curved base (possibly with the exception of the Berger space $B^{13}$ ). That is, they investigate whether a bundle $E$ over a positively curved homogeneous space up to stabilisation $E \oplus \mathbb{R}^{k}$ for some $k \geq 0$ admits a nonnegatively curved complete metric and confirm this in all cases other than $B^{13}$. In [32, Theorem 1.5] the following theorem is proved for complex bundles with stabilisation via $\mathbb{C}^{k}$.

Theorem 5.5 (González-Zibrowius) Let $G$ be a compact connected Lie group with $\pi_{1}(G)$ torsion-free, and let $H \subseteq G$ be a closed connected subgroup such that $\mathrm{rk} G-$ $\mathrm{rk} H \leq 1$. Then up to stabilisation all complex vector bundles over $G / H$ admit a complete metric of non-negative sectional curvature.

The methods in the proof are purely K-theoretic such that they extend to any base $M$ tangentially homotopic to the spaces above. This, in particular, includes homotopy spheres, since they all have stably trivial tangent bundles. (Note that not all of them admit non-negative curvature.)

Let us sketch the basic proof idea in the simplest case. We focus on complex bundles and on the case when $\operatorname{rk} G=\operatorname{rk} H$-this only holds for the even-dimensional spaces $G / H$; the odd-dimensional ones have corank rk $G-\operatorname{rk} H=1$. Consider the homogeneous space $G / H$. The first observation is that an $H$-representation $\rho$ on $\mathbb{C}^{n}$ defines a right action on $G \times \mathbb{C}^{n}$ by the associated bundle construction $((g, v), h) \mapsto$ $\left(g h, \rho\left(h^{-1} v\right)\right)$. Thus the orbit space is the total space of a complex vector bundle $E_{\rho}$, a homogeneous bundle,

$$
\mathbb{C}^{n} \hookrightarrow E_{\rho}=G \times{ }_{H} \mathbb{C}^{n} \rightarrow G / H
$$

(For example, using the isotropy representation of $H$ yields the tangent bundle.) This induces a homomorphism

$$
\alpha: R(H) \rightarrow K(G / H)
$$

from the representation ring to complex K-theory given by

$$
\rho \mapsto E_{\rho}
$$

Recall that the representation ring is given as the Grothendieck group (with respect to direct sums) of the isomorphism classes of representations of $H$. K-theory considers isomorphism classes of (complex) vector bundles over $G / H$ instead. In both cases the tensor product endows them a ring structure.

Due to O'Neill's theorem we see that whenever $G / H$ is non-negatively curved, so is $G \times{ }_{H} \mathbb{C}^{n}$. We achieve this by equipping $G \times \mathbb{C}^{n}$ with the product metric (using 
the flat metric on $\left.\mathbb{C}^{n}\right)$. Then we pass to the quotient $G \times{ }_{H} \mathbb{C}^{n}=\left(G \times \mathbb{C}^{n}\right) / H$. By O’Neill curvature may not decrease.

The proof now proceeds by showing that in any stable equivalence class of a vector bundle we may detect a homogeneous one. For this one makes use of

Theorem 5.6 (Pittie, Steinberg, [57], [59]) Let G be a compact connected Lie group such that $\pi_{1}(G)$ is torsion-free. Let $H$ be a closed connected subgroup of maximal rank. Then the homomorphism

$$
\alpha: R(H) \rightarrow K(G / H)
$$

from the complex representation ring to complex $K$-theory is surjective.

Since K-theory only sees stable classes of vector bundles, this finally amounts to the fact that in each stable class we can find a homogeneous bundle, i.e. one admitting non-negative curvature. In [32] Theorem 5.6 is extended to corank 1 groups $H \subseteq G$.

We have seen that the key ingredient in order to establish non-negative sectional curvature is the existence of $G$-equivariant structures. This existence question is examined via equivariant $\mathrm{K}$-theory. Relating the geometric description of $\mathrm{K}$ theory via vector bundles to the one as a generalised cohomology theory, also taking into account rational K-theory, and consequently examining the latter via methods from rational homotopy theory the following characterisation of the existence of $G$ equivariant bundles is provided in [10, Theorem $\mathrm{D}]$.

Theorem 5.7 (Amann-González-Zibrowius) Let $G / H$ be a closed homogeneous space, where $G$ is connected with torsion-free fundamental group, and $H \subseteq G$ is a closed connected subgroup. Then every complex vector bundle over $G / H$ carries a $G$-vector bundle structure up to stabilisation if and only if $\mathrm{rk} G-\mathrm{rk} H \leq 1$.

In fact, it was observed in [32] that the hypothesis on the rank in Theorem 5.6 can be relaxed while keeping the surjectivity of $R(H) \cong K_{G}(G / H) \rightarrow K(G / H)$, i.e. $K_{G}(G / H) \rightarrow K(G / H)$ is surjective if $\mathrm{rk} G-\mathrm{rk} H \leq 1$ (see [32, Theorem 3.6]). In [10] this implication is shown to be an equivalence by studying the induced map $K_{G}(G / H) \otimes \mathbb{Q} \rightarrow K(G / H) \otimes \mathbb{Q}$. (Here $K_{G}$ denotes $G$-equivariant K-theory analogously defined via $G$-equivariant vector bundles.)

The study of real vector bundles is quite different and more complicated. For example (see [32]), there exist spaces $G / H$ (like $\left(\mathbf{S U}(2) / \mathbb{S}^{1}\right)^{4}$ diffeomorphic to $\left.\left(\mathbb{S}^{2}\right)^{4}\right)$ as in Theorem 5.7 with rk $G-\operatorname{rk} H=0$ for which the map $\operatorname{KO}_{G}^{0}(G / H) \rightarrow$ $\mathrm{KO}^{0}(G / H)$ in real K-theory is not surjective. (Recall that real K-theory, KO-theory, is defined analogously using real vector bundles instead of complex ones.) On the other hand, it is surjective for certain spaces $G / H$ with $\operatorname{rk} G-\operatorname{rk} H$ equal to 1,2 or 3 (like any $G / H$ diffeomorphic to $\mathbb{S}^{7}, \mathbb{S}^{7} \times \mathbb{S}^{7}$, or $\mathbb{S}^{7} \times \mathbb{S}^{7} \times \mathbb{S}^{7}$, since $\mathrm{KO}^{0}(G / H)$ is trivial in this case).

We already cited the result on stable bundles over positively curved homogeneous spaces. For more general homogeneous spaces, in the spirit of the aforementioned theorems, there is (see $[10$, Theorem F]) 
Theorem 5.8 Let $G / H$ be a homogeneous space, with $G, H$ compact and connected and satisfying $\mathrm{rk} G-\mathrm{rk} H \leq 1$. Then, for every real vector bundle $E$ over $G / H$ there are integers $q>0$ and $k$ such that the Whitney sum $q E \oplus \mathbb{R}^{k}$ carries a $G$-vector bundle structure and hence the product manifold $q E \times \mathbb{R}^{k}$ admits a metric of nonnegative curvature.

Here $q E=E \oplus \stackrel{(q)}{ } q \oplus E$ denotes the $q$-fold Whitney sum.

We remark that many of these results presented for homogeneous spaces have counterparts for bundles over cohomogeneity one manifolds as worked out in [10].

\section{Conclusion, Miscellanea, and Perspectives}

We intended to illustrate that there is an interesting interplay of Riemannian manifolds with lower sectional curvature bounds, most prominently, of non-negatively and positively curved manifolds and of families of manifolds with two-sided curvature bounds with various concepts from topology. We have seen connections to

- most simple but still in general not yet computed invariants like Euler characteristics or Betti numbers.

- equivariant cohomology, which, for example, appeared in the form of localisation results connecting say Euler characteristics of fixed point sets of torus actions to the Euler characteristic of the ambient manifold. Indeed, we observed that nonnegative curvature often is studied via Lie group symmetries.

- invariants and concepts from rational homotopy theory like rational ellipticity and formality.

- index theory in the form of the $\hat{A}$-genus, twisted $\hat{A}$-genera and most prominently, the elliptic genus.

- K-theory appearing when studying the converse to the soul conjecture. This connects to equivariant K-theory and equivariant cohomology.

This is, of course, just a small selection of how curvature questions interact with topology. For example, recent progress in positive scalar curvature has brought forth various further techniques; maybe starting with the observation that certain surgeries preserve the curvature condition.

Moreover, it is an interesting problem and a subject of its own to identify properties in (equivariant) topology which have a chance to hold even on singular spaces like Alexandrov spaces-motivated by Toponogov's result these spaces define curvature bounds on geodesic length spaces via comparison triangles. In general, equivariant cohomology still has several aspects in store which have not yet found their way to geometric applications in this context. The combination of tools from Riemannian geometry and from algebraic topology always provides intriguing challenges.

As an example, let us mention that there is the interesting question when the action of a compact Lie group $G$ is equivariantly formal on a space $X$, i.e. when the LeraySerre spectral sequence of the Borel fibration $X \rightarrow X_{G} \rightarrow \mathbf{B} G$ degenerates at the $E_{2}$-term. A generalisation of this concept is provided by a Cohen-Macaulay action (cf. [28]). These concepts were investigated for transitive actions, isotropy actions or 
cohomogeneity one actions on manifolds (for example see [26], [24], [25], [16], [40], [7], [27]), and recently on cohomogeneity one Alexandrov spaces as well (see [8]). Let us end with one more conjecture (see [8, Conjecture 1.1]) relating equivariant formality to curvature on manifolds.

Conjecture 6.1 Suppose a compact connected Lie group $G$ acts isometrically on the simply-connected even-dimensional manifold $(M, g)$ of positive sectional curvature. Then the action is equivariantly formal.

We remark that this is implied by a combination of the Hopf and Bott-GroveHalperin conjectures, since the rational cohomology of a rationally elliptic space of positive Euler characteristic is concentrated in even degrees and the spectral sequence degenerates for lacunary reasons then.

Another recent strain of research comes by understanding the topology, especially the homotopy groups, of moduli spaces of metrics with certain curvature bounds. Note that the moduli space of Riemannian metrics is convex and hence contractible; however, this is far from being true once one only considers metrics with curvature bounds. Also here, active research will bring further insight.

It will be very interesting to see how this "counterpoint" of curvature versus topology keeps thriving.

Acknowledgements The author was supported by a Heisenberg fellowship and his research grant AM 342/4-1 of the German Research Foundation; he is moreover associated to the DFG Priority Programme 2026. This survey article extends a minicourse on the topic given by the author during the conference GeToPhyMa 2018 at the University of Rabat, Marocco. The author is also very grateful to Uwe Semmelmann for several helpful comments improving the presentation of the article.

Funding Note Open Access funding enabled and organized by Projekt DEAL.

Publisher's Note Springer Nature remains neutral with regard to jurisdictional claims in published maps and institutional affiliations.

Open Access This article is licensed under a Creative Commons Attribution 4.0 International License, which permits use, sharing, adaptation, distribution and reproduction in any medium or format, as long as you give appropriate credit to the original author(s) and the source, provide a link to the Creative Commons licence, and indicate if changes were made. The images or other third party material in this article are included in the article's Creative Commons licence, unless indicated otherwise in a credit line to the material. If material is not included in the article's Creative Commons licence and your intended use is not permitted by statutory regulation or exceeds the permitted use, you will need to obtain permission directly from the copyright holder. To view a copy of this licence, visit http://creativecommons.org/licenses/by/4.0/.

\section{References}

1. Amann, M.: Non-formal homogeneous spaces. Math. Z. 274(3-4), 1299-1325 (2013)

2. Amann, M.: Positive curvature, symmetry, and topology. Front. Math. China 11(5), 1099-1122 (2016)

3. Amann, M., Kennard, L.: Topological properties of positively curved manifolds with symmetry. Geom. Funct. Anal. 24(5), 1377-1405 (2014) 
4. Amann, M., Kennard, L.: Positive curvature and rational ellipticity. Algebraic Geom. Topol. 15(4), 2269-2301 (2015)

5. Amann, M., Kennard, L.: On a generalized conjecture of Hopf with symmetry. Compos. Math. 153(2), 313-322 (2017)

6. Amann, M., Kennard, L.: Positive curvature and symmetry in small dimensions. Commun. Contemp. Math. 22(06), 1950053 (2020). https://doi.org/10.1142/S0219199719500536. arXiv:1512.01302

7. Amann, M., Kollross, A.: Equivariant formality of the isotropy action on $\mathbb{Z}_{2} \oplus \mathbb{Z}_{2}$-symmetric spaces (2020). arXiv:2002.07645

8. Amann, M., Zarei, M.: On the equivariant cohomology of cohomogeneity one Alexandrov spaces (2019). arXiv:1910.06309

9. Amann, M., Ziller, W.: Geometrically formal homogeneous metrics of positive curvature. J. Geom. Anal. 26, 996-1010 (2016)

10. Amann, M., González-Álvaro, D., Zibrowius, M.: Vector bundles of non-negative curvature over cohomogeneity one manifolds (2019). arXiv:1910.05248

11. Bär, C.: Geometrically formal 4-manifolds with nonnegative sectional curvature. Commun. Anal. Geom. 23, 479-497 (2015).

12. Belegradek, I., Kapovitch, V.: Topological obstructions to nonnegative curvature. Math. Ann. 320(1), 167-190 (2001)

13. Belegradek, I., Kapovitch, V.: Obstructions to nonnegative curvature and rational homotopy theory. J. Am. Math. Soc. 16(2), 259-284 (2003) (electronic)

14. Belegradek, I., Kwasik, S., Schultz, R.: Moduli spaces of nonnegative sectional curvature and nonunique souls. J. Differ. Geom. 89(1), 49-85 (2011)

15. Breuillard, E., Green, B, Tao, T.: The structure of approximate groups. Publ. Math. IHES 116(1), 115-221 (2012)

16. Carlson, J.D., Fok, C.-K.: Equivariant formality of isotropy actions. J. Lond. Math. Soc. (2) 97(3), 470-494 (2018)

17. Cheeger, J.: Some examples of manifolds of nonnegative curvature. J. Differ. Geom. 8, 623-628 (1973)

18. Cheeger, J., Gromoll, D.: The structure of complete manifolds of nonnegative curvature. Bull. Am. Math. Soc. 74, 1147-1150 (1968)

19. Dearricott, O.: A 7-manifold with positive curvature. Duke Math. J. 158(2), 307-346 (2011)

20. Eschenburg, J.-H.: Cohomology of biquotients. Manuscr. Math. 75(2), 151-166 (1992)

21. Fang, F., Rong, X.: Curvature, diameter, homotopy groups, and cohomology rings. Duke Math. J. 107(1), 135-158 (2001)

22. Fang, F., Rong, X.: Homeomorphism classification of positively curved manifolds with almost maximal symmetry rank. Math. Ann. 332(1), 81-101 (2005)

23. Félix, Y., Halperin, S., Thomas, J.-C.: Rational Homotopy Theory. Graduate Texts in Mathematics, vol. 205. Springer, New York (2001)

24. Goertsches, O.: The equivariant cohomology of isotropy actions on symmetric spaces. Doc. Math. 17, 79-94 (2012)

25. Goertsches, O., Hagh, S., Noshari, S.: Equivariant formality of isotropy actions on homogeneous spaces defined by Lie group automorphisms. J. Pure Appl. Algebra 220(5), 2017-2028 (2016)

26. Goertsches, O., Mare, A.-L.: Non-abelian GKM theory. Math. Z. 277(1-2), 1-27 (2014)

27. Goertsches, O., Mare, A.-L.: Equivariant cohomology of cohomogeneity-one actions: the topological case. Topol. Appl. 218, 93-96 (2017)

28. Goertsches, O., Rollenske, S.: Torsion in equivariant cohomology and Cohen-Macaulay G-actions. Transform. Groups 16(4), 1063 (2011)

29. Goette, S., Kerin, M., Shankar, K.: Highly connected 7-manifolds and non-negative curvature. Ann. Math. 191(3), 829-892 (2020).

30. González-Álvaro, D.: Nonnegative curvature on stable bundles over compact rank one symmetric spaces. Adv. Math. 307, 53-71 (2017)

31. González-Álvaro, D., Radeschi, M.: A note on the Petersen-Wilhelm conjecture. Proc. Am. Math. Soc. 146, 4447-4458 (2018).

32. González-Álvaro, D., Zibrowius, M.: The stable converse soul question for positively curved homogeneous spaces. J. Differential Geom. (2017), in press. arXiv:1707.04711

33. González-Álvaro, D., Zibrowius, M.: Open manifolds with non-homeomorphic positively curved souls. Math. Proc. Camb. Philos. Soc. 169, 357-376 (2020)

34. Grosjean, J.-F., Nagy, P.-A.: On the cohomology algebra of some classes of geometrically formal manifolds. Proc. Lond. Math. Soc. (3) 98(3), 607-630 (2009) 
35. Grove, K., Halperin, S.: Dupin hypersurfaces, group actions and the double mapping cylinder. J. Differ. Geom. 26(3), 429-459 (1987)

36. Grove, K., Searle, C.: Positively curved manifolds with maximal symmetry-rank. J. Pure Appl. Algebra 91(1-3), 137-142 (1994)

37. Grove, K., Wilking, B.: A knot characterization and 1-connected nonnegatively curved 4-manifolds with circle symmetry. Geom. Topol. 18(5), 3091-3110 (2014)

38. Grove, K., Ziller, W.: Curvature and symmetry of Milnor spheres. Ann. Math. 152(1), 331-367 (2000)

39. Grove, K., Verdiani, L., Ziller, W.: An exotic $T_{1} \mathbb{S}^{4}$ with positive curvature. Geom. Funct. Anal. 21(3), 499-524 (2011)

40. Hagh, S., Noshari, S.: On the equivariant cohomology of isotropy actions. PhD thesis, Universität Marburg (2018)

41. Herrmann, M.: Homogeneous spaces, curvature and cohomology. Differ. Geom. Appl. 48, 1-10 (2016)

42. Hirzebruch, F., Slodowy, P.: Elliptic genera, involutions, and homogeneous spin manifolds. Geom. Dedic. 35(1-3), 309-343 (1990)

43. Hirzebruch, F., Berger, T., Jung, R.: Manifolds and Modular Forms. Aspects of Mathematics, vol. E20. Friedr. Vieweg \& Sohn, Braunschweig (1992). With appendices by Nils-Peter Skoruppa and by Paul Baum

44. Hsiang, W.-Y., Kleiner, B.: On the topology of positively curved 4-manifolds with symmetry. J. Differ. Geom. 29(3), 615-621 (1989)

45. Kapovitch, V., Wilking, B.: Structure of fundamental groups of manifolds with Ricci curvature bounded below (2012). arXiv:1105.5955v2

46. Kapovitch, V., Ziller, W.: Biquotients with singly generated rational cohomology. Geom. Dedic. 104, 149-160 (2004)

47. Kapovitch, V., Petrunin, A., Tuschmann, W.: Nilpotency, almost nonnegative curvature, and the gradient flow on Alexandrov spaces. Ann. Math. 171(1), 343-373 (2010)

48. Kotschick, D.: On products of harmonic forms. Duke Math. J. 107(3), 521-531 (2001)

49. Kotschick, D.: Geometric formality and non-negative scalar curvature. Pure Appl. Math. Q. 13(3), 437-451 (2017).

50. Kotschick, D., Terzić, S.: On formality of generalized symmetric spaces. Math. Proc. Camb. Philos. Soc. 134(3), 491-505 (2003)

51. Kotschick, D., Terzić, S.: Chern numbers and the geometry of partial flag manifolds. Comment. Math. Helv. 84(3), 587-616 (2009)

52. Kotschick, D., Terzić, S.: Geometric formality of homogeneous spaces and of biquotients. Pac. J. Math. 249(1), 157-176 (2011)

53. Ornea, L., Pilca, M.: Remarks on the product of harmonic forms. Pac. J. Math. 250(2), 353-363 (2011)

54. Özaydin, M., Walschap, G.: Vector bundles with no soul. Proc. Am. Math. Soc. 120(2), 565-567 (1994)

55. Petersen, P., Wilhelm, F.: An exotic sphere with positive curvature. Preprint, arXiv:0805.0812v3 [math.DG]

56. Petrunin, A., Tuschmann, W.: Diffeomorphism finiteness, positive pinching, and second homotopy. Geom. Funct. Anal. 9(4), 736-774 (1999)

57. Pittie, H.V.: Homogeneous vector bundles on homogeneous spaces. Topology 11, 199-203 (1972)

58. Rigas, A.: Geodesic spheres as generators of the homotopy groups of O, BO. J. Differ. Geom. 13(4), 527-545 (1978)

59. Steinberg, R.: On a theorem of Pittie. Topology 14, 173-177 (1975)

60. Sullivan, D.: Infinitesimal computations in topology. Publ. Math. Inst. Hautes Études Sci. 47, 269-331 (1977)

61. Totaro, B.: Cheeger manifolds and the classification of biquotients. J. Differ. Geom. 61(3), 397-451 (2002)

62. Totaro, B.: Curvature, diameter, and quotient manifolds. Math. Res. Lett. 10(2-3), 191-203 (2003)

63. Walschap, G.: Soul-preserving submersions. Mich. Math. J. 41(3), 609-617 (1994)

64. Weisskopf, N.: Positive curvature and the elliptic genus. N.Y. J. Math. 23, 193-212 (2017)

65. Wilking, B.: Torus actions on manifolds of positive sectional curvature. Acta Math. 191(2), 259-297 (2003) 
66. Ziller, W.: Examples of Riemannian manifolds with non-negative sectional curvature (2007). arXiv:math/0701389v3

67. Ziller, W.: Riemannian manifolds with positive sectional curvature (2012). arXiv:1210.4102v1

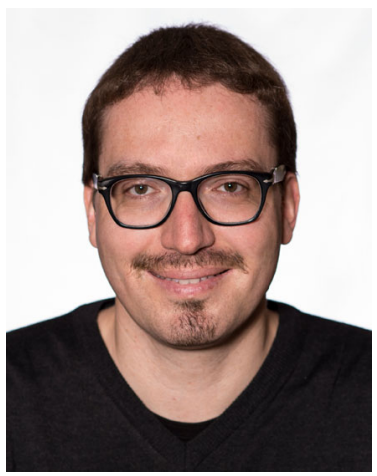

Manuel Amann received his PhD from the WWU Münster in 2009. In 2015 he made his Habilitation at the Karlsruher Institut for Technology (KIT). Since 2017 he is a Heisenberg fellow at the University of Augsburg. He received the von Kaven prize of the DFG in 2017. His research interests range from Riemannian geometry to algebraic topology, in particular involving lower curvature bounds on manifolds and singular spaces, special holonomy, rational homotopy theory, transformation groups, and equivariant cohomology. 